\title{
Rotavirus infection: An unrecognised disease in Nepal
}

\section{Pun SB}

Everest International Clinic and Research Centre, Kathmandu, Nepal

\begin{abstract}
Rotavirus is the most common cause of acute infectious gastroenteritis in young children and is associated with substantial morbidity and mortality worldwide, mostly in developing countries. The global rotavirus disease burden has prompted study on their basic research, molecular epidemiology and vaccine development. Little is known about rotavirus infection among health professionals in Nepal. This article summarises basic and clinical features, treatment and prevention, epidemiological pattern, challenges and recommendations of human rotavirus infections in Nepal.
\end{abstract}

Key words: Rotavirus, Epidemiology, Challenges and Recommendations, Nepal

$\mathrm{D}_{\mathrm{in}}^{\mathrm{i}}$ arrhoea remains one of the major causes of illness in children worldwide. It is the second leading cause of death, with, estimated $17 \%$ (1.8 million) among children under 5 globally each year (Fig.1) ${ }^{1}$. Among several microorganisms, rotavirus is the single most frequent causative agent for diarrheal episodes among infants and children in the world ${ }^{2}$. It is estimated that each year 702,000 deaths among children under 5 years of age from rotavirus gastroenteritis, mostly in developing countries ${ }^{3}$. Mortality is greatest in sub Sahara, Arica and south Asia, with about 100,000 to 150,000 (1 in 250) children die of rotavirus diarrhoea every year in India alone ${ }^{4}$. Rotavirus virtually infects all children by 3-5 years worldwide. Rotavirus mostly occurs between 6 to 23 months of age. Infants younger than 3 months of age are less likely to develop symptomatic illness, perhaps due to transplacental antibody, breast feeding or age dependent physiology $y^{5,6,7}$, while children above 5 years of age develop less severe gastroenteritis due to previous exposure that confers greater protection against subsequent disease ${ }^{8}$.

In the temperate countries, rotavirus infections occur in children during winter months ${ }^{9}$. Conversely, rotavirus infections occur throughout the year in developing countries, but disease is more commonly observed during cooler and drier months ${ }^{10}$.

There is no difference in the rates of rotavirus illness among children between developed and developing countries, indicating that good hygiene and clean water supplies do not prevent the disease ${ }^{2}$. No antiviral therapies are available and treatment of rotavirus diarrhoea remains only fluids and electrolytes replacement. To reduce substantial rotavirus disease burden, safe and effective rotavirus vaccines are urgently needed. Rotavirus vaccines would have significant impact on reducing rotavirus incidence, especially in sub Sahara and south Asia, where the vaccine is in greatest need.

\section{Virus structure and classification}

Rotavirus is $70 \mathrm{~nm}$ icosahedral, non-enveloped virus that belongs to the family Reoviridae. The virus is composed of three protein shells, consisting of a core, an inner and an outer capsid which make wheel-shaped structure (from the Latin rota, meaning "wheel”) (Fig 2). It has a genome of 11 double-stranded RNA segments, which encode six structural viral proteins (VP1-VP4, VP6, VP7) and six non structural viral proteins (NSP1NSP6) ${ }^{11}$. In infected cells, non structural proteins are produced and are not incorporated into the mature virions, thus known as non structural proteins. Each viral genome codes for a single protein except genome segment 11 that encodes two protein called NSP5 and NSP6. VP1, VP2 and VP3 form the core of the virion and are involved in genome replication and package ${ }^{11}$. The outer most capsid is made up of VP7 and VP4 proteins. They induce virus neutralization antibodies. VP4 is cleaved by an intestinal lumen protease (trypsin) into VP5* and VP8*11. This cleavage of VP4 enhances the infectivity of rotavirus. VP6 is the most abundant protein in the virus which forms the inner capsid and used in diagnostic assay for the detection of antigen. Group and subgroup is based on VP6 protein.

Correspondence

Dr. Sher Bahadur Pun

Everest International Clinical and Research Centre

Kathmandu, Nepal

E-mail: drsherbdr@yahoo.com 
Seven distinct groups (A-G) of rotaviruses have been described ${ }^{11}$. Group A, B and C rotaviruses have been found in both humans and animals whereas groups $\mathrm{D}, \mathrm{E}, \mathrm{F}$ and $\mathrm{G}$ have been found only in animals to date. Group B rotaviruses also called adult diarrhoea rotavirus (ADRV); have been found large outbreaks of severe gastroenteritis in adults primarily in china ${ }^{12}$. Group B rotaviruses have been also reported from India and Bangladesh ${ }^{13,14}$. Group $\mathrm{C}$ rotaviruses have been found sporadic cases and outbreaks of diarrhoea in children. Among three human rotaviruses (A, B and C), group A rotaviruses have been established as causing several diarrheal diseases in infants and young children worldwide ${ }^{11}$.

Group A rotaviruses are classified into serotypes on the basis of outer capsid proteins VP7 (G serotypes) and VP4 (P serotypes $)^{11}$. The $\mathrm{G}$ serotype defined by glycoprotein and the $P$ serotype defined by protease- sensitive protein. On the basis of VP7, 19 different G serotypes have been known so far $^{15}$. P serotypes that are referred to by their numbers (eg.P1, P2), are difficult to characterise by traditional methods of virus neutralisation; therefore, molecular methods based on sequencing are widely used and are indicated by a number in square brackets (eg. P[1], P[2]). Currently 29 P genotypes are known ${ }^{15}$. $G$ and $P$ serotypes have been adopted to define rotavirus serotype. G1, G3 and G4 associated with P [8]; and G2 with $\mathrm{P}[4]$ represented over $88 \%$ of all rotavirus strains worldwide ${ }^{16}$. G and P antigens segregate independently and interspecies transmission between humans and animals are common ${ }^{17}$. Thus unusual combinations of $P$ and $G$ have been observed over the past several years ${ }^{18}$.

\section{Clinical features and diagnostic methods}

Rotavirus infection produces in a various severity of gastroenteritis ranging from subclinical to severe lifethreatening diarrhoea with dehydration. Rotavirus spreads directly via the faecal-oral route. The incubation period for rotavirus ranges from 1 to 3 days. Vomiting, Fever and dehydration are the major clinical features of rotavirus infection in children. In one study done in Eastern Nepal made a comparison between the clinical manifestations of 62 patients hospitalised with rotavirus diarrhoea and 98 patients hospitalised with non rotavirus diarrhoea $^{19}$. Fever was seen in both groups. However, vomiting and dehydration were seen predominantly in rotavirus positive group than the rotavirus negative group. Thus more rotavirus positive group needed hospitalization as compared to rotavirus negative group. These clinical features attributable to rotavirus diarrhoea in children were comparable to findings in other studies ${ }^{20}$. Symptoms of acute infection can persist for 3 to 8 days. The period may be longer in immunecompromised patients ${ }^{21}$.
The clinical features of rotavirus infections are not sufficient to identify rotavirus for diagnosis. Thus diagnosis requires viral antigens or viral nucleic acid detection. Although Electron Microscope (EM) continues to be the important in the diagnosis of rotaviral diseases, other techniques such as Enzyme-linked immunosorbent assay (ELISA), Electropherotyping (using Polyacrylamide Gel Electrophoresis, PAGE) and reverse transcription -polymerase chain reaction (RTPCR) are widely used to detect and characterize rotavirus strains. Among these methods, ELISA has become the method of choice for screening rotavirus in many laboratories, since it is highly sensitive, provide rapid results and widely available. Electropherotype provides specific information for identifying single strain and strain diversity among circulating rotavirus strain in a given place. Thus it has been used for epidemiological studies worldwide. RT-PCR is used for identifying rotavirus genotype based on nucleotides. This method is useful for serotype-specific genotyping rapidly. PCR product further can be used for sequencing, enabling to detect genetic diversity and unusual strain among rotaviruses.

\section{Treatment and Prevention}

Intravenous fluid administration has been used for treating severe rotavirus gastroenteritis. Oral rehydration solutions (ORS) are highly effective in the treatment of dehydration caused by rotavirus. However, ORS does correct some and no dehydration and once severe dehydration is corrected and during maintenance phase. When the patient is severely dehydrated or in shock, intravenous fluids must be given. There are no antiviral agents available for the treatment of rotavirus infection.

Global burden of rotavirus disease has made development of preventive strategies as a priority by World Health Organization ${ }^{22}$. Vaccines were recognized as an effective intervention to prevent severe rotavirus gastroenteritis. Currently two rotavirus vaccines RotaTeq and Rotarix have been licensed in more than 100 countries $^{23}$. The realist goal of these vaccines is to duplicate the degree of protection against diseases following natural infections that provide protection against severe rotavirus gastroenteritis leading to dehydration and hospitalisation or death.

\section{Rotavirus Epidemiology in Nepal}

Until recently, few studies were carried out on rotavirus disease in Nepal. Most of these studies were conducted for a short period of time without molecular study. Molecular characterisation of rotavirus gastroenteritis in Nepal was initiated in $2003^{24}$. Thus molecular knowledge on rotavirus strains in Nepal was not well known. 
However, 4-year study on molecular epidemiology between 2003 and 2007 reflect rotavirus strains circulating in $\mathrm{Nepal}^{24,25,26}$. The studies have documented huge diversity of rotavirus strains circulating in Nepal. In addition, a significant number of unusual strains were detected among rotavirus strains. G1 and G2 have been identified as the major causes of diarrhoea in humans, whereas G11 and G12 were unusual strains emerging in Nepal.

Studies published on rotavirus infection from 1999 to 2007 showed rotavirus positivity rates ranged from 17 to 39 percent (median $31.8 \%$ ) in among all hospitalised children less than 5 years ${ }^{19,24,25,26,27}$ (Fig.3). Rotavirus infections have been found more than $85 \%$ in children by the age of 3 years, whereas $2.6 \%$ below 3 months of age group ${ }^{26}$. However, only $55 \%$ of rotavirus associated hospitalizations occurred among children in the first year of life. This is in contrast to other findings from low income countries, where $\sim 80 \%$ rotavirus associated hospitalization occurred among children during the first year of life ${ }^{28}$. Rotavirus infections have been detected mostly in the low temperature and dry season, peaking in January, whereas lower number in the hot and rainy seasons between June and September in Nepal.

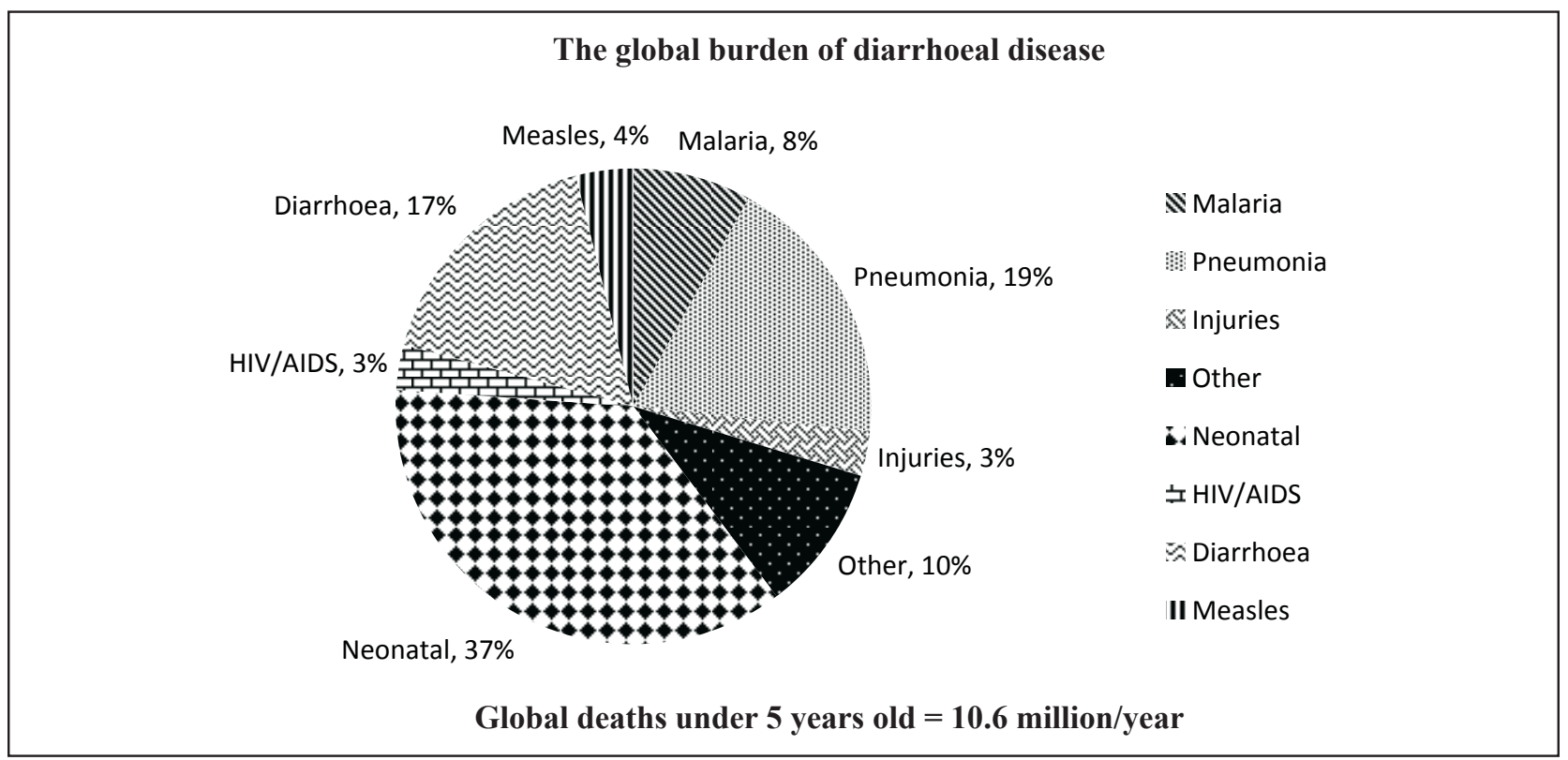

Fig 1: Global deaths of children under 5 years old and 2007

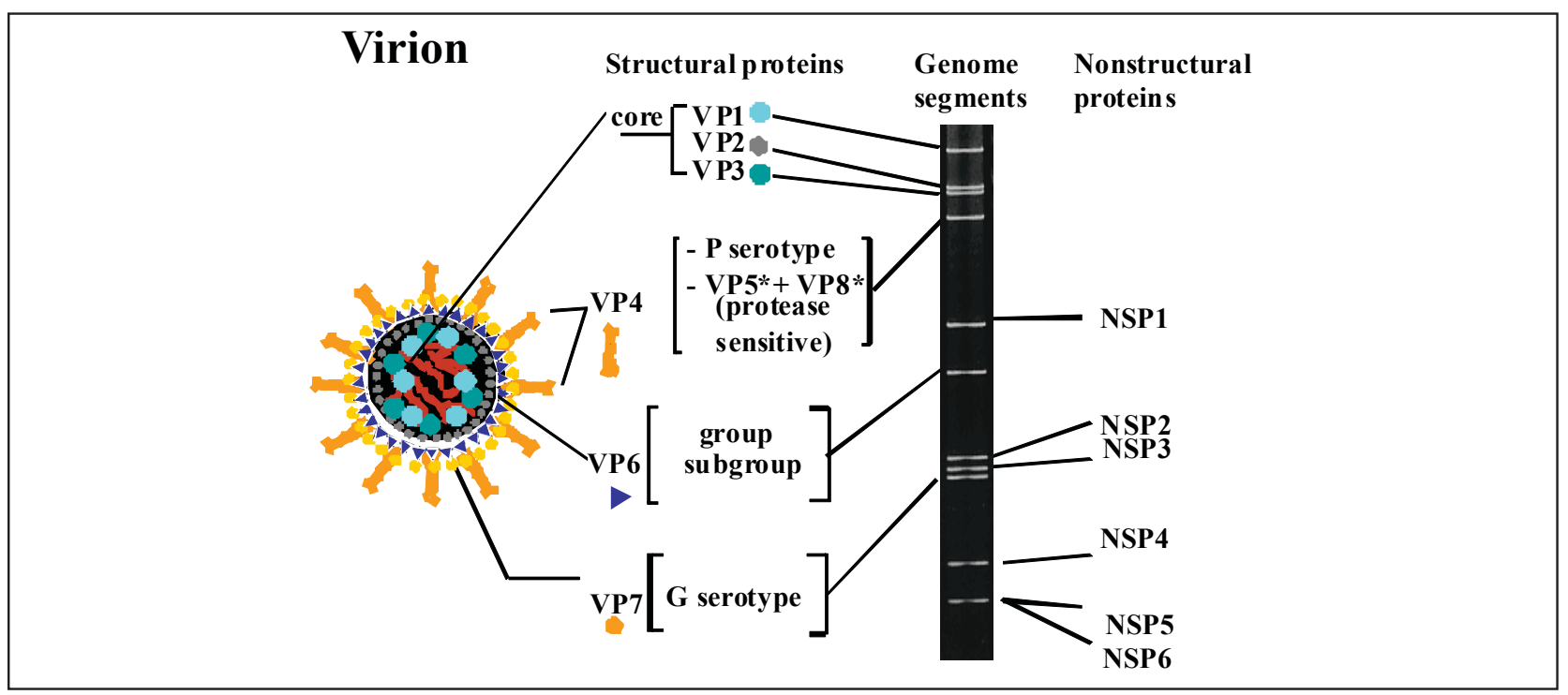

Fig 2: A Schematic diagram showing relationships among proteins (structural and non-structural) and the genomes segments which encodes these viral proteins. 


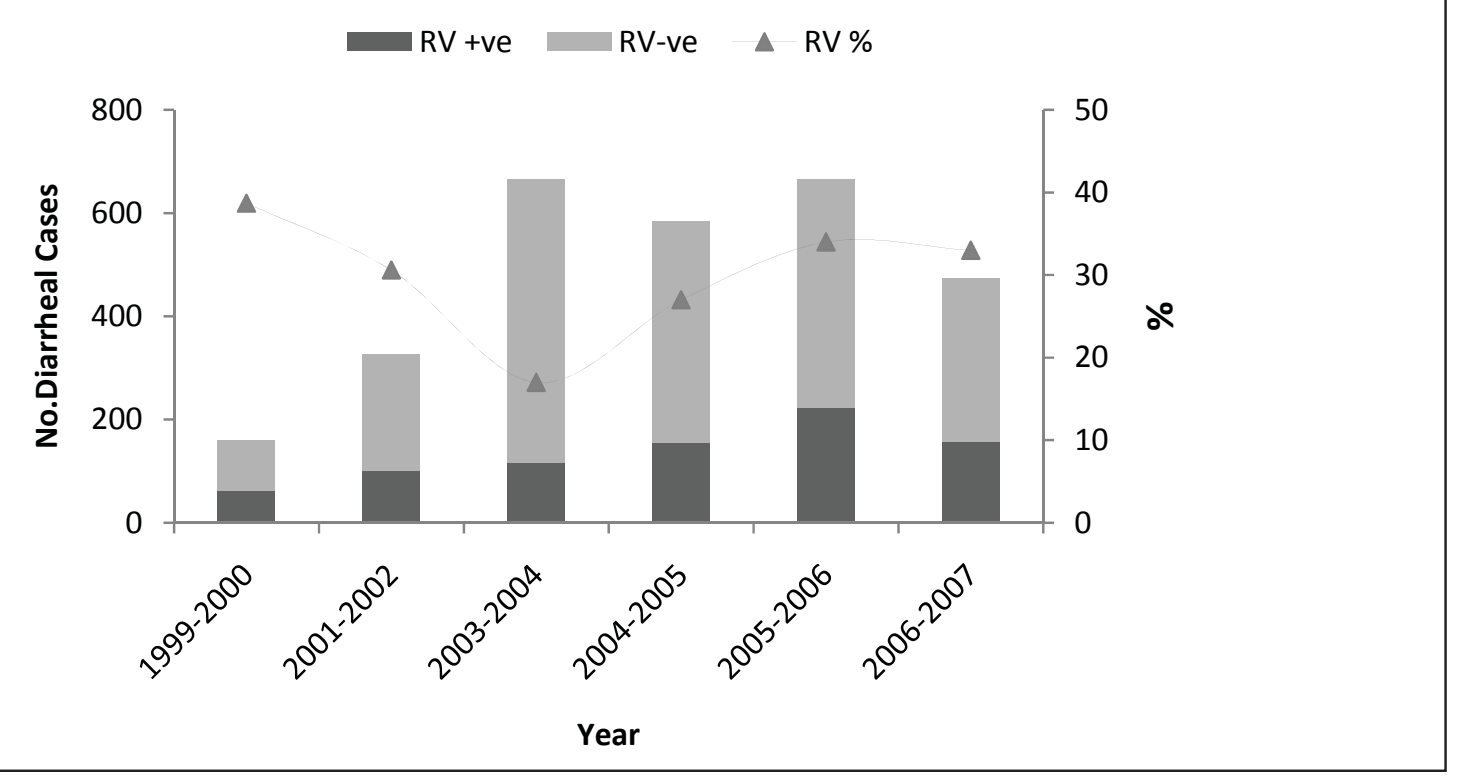

Fig 3: Yearly distribution of Rotavirus (RV) infection among hospitalized diarrhoeal children under 5 in Nepal, between 1999 and 2007

\section{Challenges and Recommendations}

Several challenges lie ahead of vaccine introduction in Nepal. Diarrhoeal diseases have long been the major public health problem, with estimated 45,000 annual deaths due to diarrhoea among children less than 5 years of age in $\mathrm{Nepal}^{29}$. Although rotavirus infections have been recognised as the leading cause of acute gastroenteritis among young children, only a few studies have been carried out on rotavirus diarrhoea in Nepal. Physicians do not seek rotavirus as the aetiology agent of gastroenteritis, since it does not alter treatment with rehydration therapy. Thus newly available rotavirus vaccines may not receive significant attention it deserves from decision makers and paediatricians due to the absence of knowledge about the local burden of diseases. Nationwide active rotavirus surveillance network, therefore, should be established in order to determine national rotavirus disease burden, especially beyond urban areas, where rotavirus disease burden could be greater and unusual rotavirus strains may be emerging in human populations due to close physical contact with livestock. Unusual rotavirus strains could challenge currently available vaccines, since these vaccines are primarily directed against prevalent rotavirus serotypes and protection against unusual strains have not yet fully determined.

Rotashield, the first licensed rotavirus vaccine, was withdrawal from the market in less than a year in the United States due to its association with intussusceptions among vaccine recipients ${ }^{30}$. Thus to ensure the risk of intussusception from future vaccines would be minimised, the two currently available vaccines
(RotaTeq and Rotarix), were evaluated with samples size exceed of 60,000 that showed no association with intussusception $^{31,32}$. However, either of the vaccine has insufficient safety baseline data with respect to intussusception in the least developed countries of sub-Saharan and south Asia, where the disease burden is greatest. Thus anticipating rotavirus vaccines in Nepal, study on intussusception among children would be crucial before and after the vaccination to compare vaccine-associated intussusception among recipients.

The question then arises as to immunogenicity and vaccine efficacy in Nepal. Currently available rotavirus vaccines have shown to be efficacious in clinical trials in the Americas and Europe, but need to be proven in poor resources countries. Although some studies have evaluated vaccine efficacy in low income countries in Africa and $\mathrm{Asia}^{33,34}$, more studies will be required, where malnutrition, co infections and unusual rotavirus strains are prevalent. Unusual strains such as G12, which was circulating in Nepal between 2003 and 200724,25,26, could be a potential challenge to the currently available vaccines.

Financial sources will be the key issue in introducing rotavirus vaccine in Nepal. Although the Global Alliance for Vaccines and Immunizations (GAVI) has pledged for financial support to accelerate the introduction of rotavirus vaccine in developing countries, financial long term support will be needed to ensure a continuous vaccine supply in future. 


\section{Concluding Remarks}

In 1973 Bishop and colleagues have discovered rotavirus in children with diarrhoea ${ }^{35}$. Since then rotavirus infection has been established as a major cause of gastroenteritis in infants and children worldwide. Rotavirus has been shown to cause 39\% (range 29-45\%) childhood diarrhoea worldwide ${ }^{36}$. Immune response that develops after primary infection is protective against severe gastroenteritis on subsequent reinfection ${ }^{8}$. On the basis of this simple fact, two live attenuated rotavirus vaccines (RotaTeq and Rotarix) have been developed and are now licensed more than 100 countries $^{23}$. Although there is no significant difference in the incidence of rotavirus infection between developed and developing countries, children in developing countries have higher mortality rates than in developed countries. Thus these life saving rotavirus vaccines are urgently needed in sub Saharan and south Asia, where rotavirus disease burden is greatest.

Limited available data demonstrated that the leading cause of hospitalisation due to gastroenteritis among young children is associated with rotavirus infection in Nepal. According to the Annual Report 2006/2007(Department of Health Services, Nepal), the incidence of diarrhoea has been drastically decreased due to improved sanitation and drinking water. However, winter diarrhoea did not reduce significantly, which could be attributed to rotavirus infection ${ }^{37}$. The time, therefore, has come to estimate accurate rotavirus disease burden, pre-licensure clinical trials and rotavirus vaccine introduction into national immunisation schedules in Nepal. In addition poor health care facilities, insufficient health care professional and difficult geographical terrain are the major obstacles in Nepal to treat severe gastroenteritis patients on time, which often lead to severe dehydration, hospitalisations and/ death. In order to overcome with these hurdles, rotavirus vaccination is only the option likely to have a significant impact in preventing infants and young children from severe rotavirus gastroenteritis, and will be helpful to Nepal government in achieving the Millennium Development Goals (MDG) by reducing child mortality by two-third by the year 2015 .

Acknowledgement: I am grateful to Osamu Nakagomi for reviewing the manuscript.

\section{References}

1. Bryce J, Boschi -Pinto C, Shibuya K, Black RE. WHO estimates of the cause of death in children. Lancet. 2005;365:1147-52.

2. Kapikian AZ. Viral gastroenteritis. JAMA. 1993;269:627-30.

3. Cunliffe NA, Nakagomi O. A critical time for rotavirus vaccines: a review. Expert Rev Vaccines. 2005;4(4):521-32.

4. Jain V, Prashar UD, Glass RI, Bhan MK. Epidemiology of rotavirus in India. Indian $\mathrm{J}$ pediatr. 2001;68:855-62.

5. Rojas AM, Boher Y, Guntinas MJ, Perez Schael I. Homotypic immune response to primary infection with rotavirus serotype G1. J Virol. 1995;47:404-9.

6. Rimer HC, Wasserman SS, Flores J, Pichichero ME, Losonsky GA. Rotavirus-specific breast milk antibody in two populations and possible correlates of interference with rhesus rotavirus vaccine seroconversion. $\mathrm{J}$ Infec Dis. 1992;165:826-30.

7. Bass DM, Baylor M, Broome R, Greenberg HB. Molecular basis of age-dependent gastric inactivation of rhesus rotavirus in the mouse. $\mathrm{J}$ Clin Invest. 1992;89:1741-5.

8. Velazquez FR, Matson DO, Calva JJ, Guerrero L, Marrow AL, Carter-Campbell L, et al. Rotavirus Infections in infants as protection against subsequent infections. N Engl J Med. 1996;335(14):1022-8.

9. Kapikian AZ, Kim HW, Wyatt RG, Cline WL, Arrobio JO, Brandt CD, et al. Human reoviruslike agent as the major pathogen associated with "winter" gastroenteritis in hospitalized infants and young children. N Engl J Med. 1976;294:965-72.

10. Cunliffe NA, Kigore PE, Bresee JS, Luo N, Hart CA, Glass RI. Epidemiology of rotavirus diarrhea in Africa: a review to assess the need for rotavirus immunization. Bull WHO. 1998;76:525-37.

11. Estes MK. Rotaviruses and their replication. In: Knipe DM, Howley PM, eds. Fields virology. Philadelphia: Lippincott Williams \& Wilkins;2001.p.1747-85.

12. Hung $T$, Chen G, Wang C, Zingyi C, Tungxin C, Wei Wei $\mathrm{Y}$, et al. Rotavirus-like agent in adult non-bacterial diarrhoea in China. Lancet.1983;2:1078-9.

13. Kobayashi N., Naik TN, Kusuhara Y, Krishnan T, Sen A, Bhattacharya SK, et al. Sequence analysis of genes encoding structural and nonstructural proteins of a Human group B rotavirus detected in Calcutta India. J Med Virol. 2001; 64 (4): 583-8.

14. Alam MM, Kobayashi N, Ishino M, Wakuda M, Ahmed MS, Ahmed MU, et al. Genetic analysis of an ADRV-N-like novel rotavirus strain B219 detected in a sporadic case of adult diarrhea in Bangladesh. Arch Virol.2007;152:199-208. 
15. Greenberg H, Estes M. Rotaviruses: From Pathogenesis to Vaccination. Gastroenterology. 2009;136:1939-51.

16. Santos N, Hoshino Y. Global distribution of rotavirus serotypes/genotypes and its implication for the development and implementation of an effective rotavirus vaccine. Rev Med Virol. 2005;15:29-56.

17. Shif I, Iizuka M, Silberstein I, Mendelson E, Nakagomi O. Rotaviruses belonging to the AU1 genogroup recovered from Israeli infants with diarrhea. Arch Virol.1994;138:357- 64.

18. Ramachandra M, Das BK, Vij A, Kumar R, Bhambal SS, Keshari N, et al. Unusual Diversity of Human Rotavirus G and P Genotypes in India. J Clin Microbiol. 1996;34:436-9.

19. Shariff M, Deb M, Singh R. A study of diarrhoea among children in Eastern Nepal with special reference to Rotavirus. Indian J Med Microbiol. 2003;21(2):87-90.

20. Karadag A, Acikgoz ZC, Avci Z, Catal F, Gocer $\mathrm{S}$, Gamberzade $\mathrm{S}$, et al. Childhood diarrhoea in Ankara, Turkey: Epidemiological and clinical features of rotavirus-positive versus rotavirusnegative cases. Scand J Infect Dis.2005;37:26975.

21. Oishi I., Kimura T., Murakami T., Haruki K., Seto Y., Minekawa Y. Funamoto H. (1991) Serial observations of chronic rotavirus infection in an immunodeficient child. Microbiol. Immunol.1991;35:953-61.

22. World Health Organization. Rotavirus Vaccines, an update. Wkly Epidemiol Rec.2003;78:2-3.

23. Nakagomi O, Cunliffe NA. Rotavirus vaccines: entering a new stage of deployment. Curr Opin Infect Dis. 2007;20:501-7.

24. Uchida R, Pandey DV, Sherchand JB, Ahmed K, Yokoo M, Nakagomi T, et al. Molecular Epidemiology of Rotavirus Diarrhea among Children and Adults in Nepal: Detection of G12 strains with $\mathrm{P}[6]$ or $\mathrm{P}[8]$ and a G11P[25] Strain. J Clin Microbiol. 2006;44: 3499-505.

25. Pun SB, Nakagomi T, Sherchand JB, Pandey BD, Cuevas LE, Cunliffe NA, et al. Nakagomi O Detection of G12 Human Rotaviruses in Nepal. Emerg Infect Dis. 2007;13:482-4.

26. Sherchand JB, Nakagomi O, Dove W, Nakagomi T, Yokoo M, Pandey BD, et al. Molecular Epidemiology of Rotavirus Diarrhea among Children Aged $<5$ Years in Nepal: Predominance of Emergent G12 Strains during 2 Years. J Infect Dis. 2009; 200:S187.
27. Sherchand JB, Haruki K. Rotavirus Diarrhea in Children and Animal of Urban and Rural Nepal. Journal Nepal Health Research Council. 2004; 2:5-8.

28. Bresee JS, Hummelman E, Nelson EAS. Glass RI. Rotavirus in Asia: The value of Surveillance for Informing Decisions about the Introduction of New Vaccines. J Infect Dis. 2005; 192:S1-5.

29. Jha N, Singh R, Baral D. Knowledge, attitude and practices of mothers regarding home management of acute diarrhea in Sunsari, Nepal. Nepal Med Coll J. 2006; 8(1): 27-30.

30. Murphy TV, Garguillo PM, Massoudi MS, Nelson DB, Jumaan AO, Okoro CA et al. Intussusception among infants given an oral rotavirus vaccine. N Engl J Med. 2001; 344:56472.

31. Ruiz-Palacios GM, Perez-Schael I, Velazquez FR, Abate F, Breuer T, Clemens SC et al. Safety and efficacy of an attenuated vaccine against severe rotavirus gastroenteritis. N Engl J Med.2006; 354: 11-22.

32. Vesikari T, Matson DO, Dennehy P, Van Damme P, Santosham M, Rodriguez Z et al. Safety and efficacy of a pentavalent human-bovine (WC3) reassortant rotavirus vaccine. N Engl J Med.2006; 354: 23-33.

33. Steele AD, De Vos B, Tumbo J, Reynders J, Scholtz F, Bos P, et al. Co-administration study in South African infants of a live-attenuated oral human rotavirus vaccine (RIX4414) and Polio vaccines. Vaccine. 2008; doi: 10.1016/ j.vaccine.2008.08.034

34. Zaman K, Sack DA, Yunus M, Arifeen SE, Podder G, Azim T, Luby S, Breiman RF, Neuzil K, Datta SK, Delem A, Suryakiran PV, Block $\mathrm{HL}$, and the Bangladeshi Rotavirus Vaccine study group. Successful co-administration of a human rotavirus and oral polio vaccines in Bangladeshi infants in a 2-dose schedule at 12 and 16 weeks of age. Vaccine.2009; 27:133339

35. Bishop RF, Davidson GP, Holmes IH, and Ruck BJ. Virus particles in epithelium cells of duodenal mucosa from children with viral gastroenteritis. Lancet. 1973;2:1281-83

36. Parashar UD, Gibson JC, Bresee JS, and Glass RI. Rotavirus and Severe Childhood Diarrhea. Emerg Infect Dis. 2006; 12: 304-6

37. Department of Health Services, Ministry of Health, Nepal. Annual Report (2006/2007). Kathmandu: Department of Health Services, Ministry of Health, Nepal; 2007.p. 68-75. 